\title{
Protecting Participants in Thought Experiments: The Role of the Research Ethics Committee
}

\author{
David Shaw
}

Received: 23 October 2017 / Accepted: 24 October 2017 /Published online: 8 December 2017

(C) Journal of Bioethical Inquiry Pty Ltd. 2017

\begin{abstract}
A fat man stands upon a bridge. A runaway train is about to pass under the bridge and kill five people who are tied to the tracks half a mile down the line. You stand behind the fat man and could push him in front of the train. He is so fat that the train will definitely stop before it hits the people. Pushing him will save five lives but he will not survive. Do you do it? ${ }^{1}$
\end{abstract}

Imagine if a research ethics committee (REC) was asked to grant permission for a study actually setting up this experiment in real life to investigate whether people would push the man. They would never approve such a study because it would involve the deaths of participants. But experiments like this are used all the time- - as thought experiments. Unlike clinical trials, or psychological studies, or even qualitative interviews and surveys, thought experiments do not require ethical approval. But do their participants not deserve protection too? Thought experiments are published in theoretical research articles as well as being used in experimental philosophy research where members of the public are asked to consider such scenarios. As such, the examples used in such experiments could have unethical effects.

$\overline{1}$ Adapted from Thomson 1976

D. Shaw $(\bowtie)$

Institute for Biomedical Ethics, University of Basel,

Bernoulistrassse 28, 4056 Basel, Switzerland, and Department of Health, Ethics and Society, CAPHRI Research Institute Maastricht University, Maastricht, The Netherlands

e-mail: david.shaw@unibas.ch
If a REC was reviewing the scenario above, even in the context of a thought experiment, one can imagine some of the comments made by members (this is itself a thought experiment, of course: we assume that the researcher attends the meeting, as is normal for NHS ethics committees). The first comment is: "Why does he need to be fat? That's offensive to overweight and obese people. This inclusion criterion is arbitrary." The researcher replies that it's not intended to be offensive, but only a large heavy mass will stop the train so the parameters of the thought experiment require that he be fat. Another member of the committees says "but it doesn't have to be a fat person, it just has to be a person! Any train driver would stop immediately if he hits someone." To this the researcher replies that it's not a passenger train but a runaway goods train, thereby answering the next question which was going to be about the risk to passengers if the train derailed. In response the chair points out that the type of train and the fact that it doesn't carry passengers should be specified in the protocol.

A lay member of the committee is less concerned about the fat man (thinking though not saying "must eat too much junk food, probably not long to live anyway") but very concerned about the five people tied to the tracks. How were the five people selected? When the researcher (a philosopher) tries to explain that that's not relevant to the experiment the lay member becomes irate. "Of course it's relevant! What if they're all murderers? Maybe then you shouldn't push the differently sized gentleman!" The researcher replies that we must assume that they are all innocent people alike in all 
relevant respects. The statistician asks whether it's really necessary to have five people when two would be sufficient to make the point. The philosopher sniffily suggests that the REC isn't meant to comment on design issues, only ethical ones, to which the chair replies that bad design is itself an ethical issue - all the more so when participants' lives are at risk from a speeding train and including more could be superfluous. The researcher replies that five was chosen because one on one wouldn't be enough and ten would be extravagant. The chair agrees, pointing out that the Declaration of Hellsinkhole (named after the location of an infamous thought experiment involving demons and an unfortunate geological accident) stipulates that no more people should be sacrificed in a thought experiment than is strictly necessary for philosophical power. Another lay member reminds the applicant that research should be of societal relevance and he doesn't see any in this experiment. The researcher replies that it's important to know the circumstances under which it's deemed acceptable to kill one person to save many. The chair says "I wonder that a lot chairing this committee."

On balance, the committee decides to approve the thought experiment because the benefits to science and to those asked to think about it (in terms of clarifying moral principles) outweigh the potential harms to the fictional participants. The chair moves on to the next research proposal. It concerns connecting a kidnapped person to an unconscious violinist with kidney failure for the purpose of dialysis (for the purposes of a thought experiment about abortion)(Foot 1978).

Acknowledgements Thanks to Bart Penders for helpful discussion about this paper.

\section{Compliance with ethical standards}

Competing Interests No conflicts of interest to declare, except that I once chaired a research ethics committee and have designed lots of thought experiments.

\section{References}

Foot, P. 1978. The problem of abortion and the doctrine of double effect. In Virtues and Vices. Oxford: Blackwell.

Thomson, J.J. 1976. Killing, letting die, and the trolley problem. The Monist 59(2): 204-217. 